\title{
Nanoemulsion loaded with lycobetaine-oleic acid ionic complex: physicochemical characteristics, in vitro, in vivo evaluation, and antitumor activity
}

This article was published in the following Dove Press journal:

International Journal of Nanomedicine

18 May 2013

Number of times this article has been viewed

\section{Hui Zhao \\ Hua Lu \\ Tao Gong \\ Zhirong Zhang}

Key Laboratory of Drug Targeting, Ministry of Education, Sichuan University, Chengdu, People's

Republic of China
Correspondence: Tao Gong

Key Laboratory of Drug Targeting,

Ministry of Education, West China

School of Pharmacy, Sichuan University,

Number 17, Section 3, Southern

Renmin Road, Chengdu 61004I,

People's Republic of China

Tel +8602885501615

Email gongtaoy@126.com

Zhirong Zhang

Key Laboratory of Drug Targeting,

Ministry of Education, West China

School of Pharmacy, Sichuan University,

Number 17, Section 3, Southern

Renmin Road, Chengdu 61004I,

People's Republic of China

Tel +860288550 I6I5

Email zrzzl@vip.sina.com
Background: Intravenous injection of lycobetaine was found to show significant cytotoxic activity against (inter alia) Lewis lung carcinoma, but its therapeutic use is largely limited due to an extremely short half-life in blood. This study aimed at developing a novel lipid nanocarrierbased formulation for lycobetaine delivery. The formulation is feasible for scale-up production, exhibiting good parenteral acceptability and improved circulation characteristics.

Methods: To enhance its lipophilicity, oleic acid was selected to form ionic complexes with lycobetaine (LBT). The nanoemulsion loaded with LBT-oleic acid complex (LBT-OA-nanoemulsion) and PEGylated LBT-OA-nanoemulsion (NE) (LBT-OA-PEG-NE) were prepared by a simple high-pressure homogenization method.

Results: A high-encapsulation efficiency of around $97.32 \% \pm 2.09 \%$ was obtained for LBT-OA-PEG-NE under optimized conditions. Furthermore, the in vivo pharmacokinetics and biodistribution of LBT-OA-NE, LBT-OA-PEG-NE, and free LBT were studied in rats. Free LBT and LBT-OA-PEG-NE displayed $\mathrm{AUC}_{0-10 \mathrm{~h}}$ (area under the concentration-time curve from 0 to 10 hours) of $112.99 \mathrm{mg} / \mathrm{L}^{*}$ minute and $3452.09 \mathrm{mg} / \mathrm{L}^{*}$ minute via intravenous administration $(P<0.005)$, respectively. Moreover, LBT-OA-PEG-NE showed significantly lower LBT concentration in the heart, liver, and kidney, while achieving higher concentration of LBT in the lung when compared to free LBT at the same time $(P<0.005)$. The LBT-OAPEG-NE exhibited higher growth inhibitory effect and longer survival time than free LBT in both heterotopic and lung metastatic tumor models.

Conclusion: These results demonstrated that LBT-OA-PEG-NE is an attractive parenteral formulation for cancer therapy.

Keywords: lycobetaine, oleic acid, polyethylene glycol, nanoemulsion, in vivo studies, antitumor activity

\section{Introduction}

Lycobetaine (ungeremine; AT-1840; LBT) (Figure 1) is a quaternary phenanthridinium alkaloid from Lycoris radiata of the Amaryllidaceae family. ${ }^{1}$ Intravenous injection of this alkaloid was found to show significant cytotoxic activity against (inter alia) Lewis lung carcinoma in lab animals. ${ }^{2,3}$ Lycobetaine was shown to act as a topoisomerase inhibitor which inhibits both topoisomerases I and II. ${ }^{4,5}$ Lycobetaine (LBT) was demonstrated to have a curative effect, low toxicity, no myelosuppression, when studied in the $1980 \mathrm{~s}$ extensively ${ }^{6,7}$ However, its therapeutic use is largely limited, due to an extremely short half-life in the blood of about 30 seconds. ${ }^{7,8}$ Moreover, no reported drug delivery systems were found for LBT delivery. Therefore, a novel drug-delivery system was developed for LBT that could extend the circulation time of the drug in vivo. 


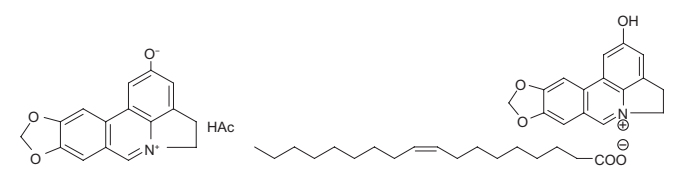

Figure I Chemical structures of lycobetaine and the lycobetaine-oleic acid ionic complex.

Recently, among various lipid nanoparticles, increasing attention has been focused on nanoemulsions as colloidal drug-carrier systems, avoiding some disadvantages of nanoparticles, microemulsions, niosomes, liposomes, and simultaneously combining their advantages, such as: (1) stability against inherent creaming, flocculation, coalescence, and sedimentation; (2) easily prepared; (3) solubilization properties; (4) ability not to damage healthy human and animal cells; (5) easy to produce; (6) and small droplet size. ${ }^{9}$ Lipid nanoparticles displayed advantages, such as prolonged release, high drug-loading capacity, and drug targeting, ${ }^{10}$ but they also create some imperfections such as a weak uptake capacity for the drug. ${ }^{11}$ Nanoemulsions also have several biological applications: as a carrier for targeted drug delivery; as a prophylactic in bioterrorism attacks; as a nontoxic disinfectant cleaner; in improved oral delivery of poorly soluble drugs as a vehicle for transdermal. ${ }^{12}$ These unique characteristics of nanoemulsions (NE) highlight them as suitable drug carriers with improved targeting properties. It has been demonstrated that with the help of NEs as a delivery system, the retention time of a drug in the body can be increased, thereby reducing the amount of drug required for a therapeutic treatment. Thus, NEs can help achieve drug targeting while protecting the drug from degradation/elimination in vivo. The components of NE, such as lecithin, soybean oil (injectable grade), and structured lipids (injectable grade), have been clinically available for several decades and are currently used in many parenteral formulations. Therefore, NE are much more suitable for encapsulating LBT than other nanocarriers. Short half-life drugs used in clinics are administered generally via intravenous infusion. Surface modification of nanocarriers with amphiphilic polymer polyethylene glycol is thought to provide steric stabilization for the particles. Polymer polyethylene glycol chains offer an "invisibility" to the nanoparticles. This was reflected in a reduced recognition of the mononuclear phagocyte system and an extended time of circulation. ${ }^{13}$ Therefore, polymer polyethylene glycolylated NE is designed and developed for the parenteral delivery of LBT.

In this study, we aimed to develop biocompatible nanocarriers for LBT delivery. To enhance the lipophilicity of
LBT necessary for encapsulation, oleic acid was selected as the lipophilic complexing agent to form LBT-oleic acid ionic complex (LBT-OA). LBT-oleic acid (OA) complex loaded NE (LBT-OA-NE) and PEGylated LBT-OA-NE (LBT-OA-PEG-NE) were prepared by a simple high pressure homogenization method. Both LBT-OA-NE and LBTOA-PEG-NE nanocarriers displayed high encapsulation ratio and excellent size distribution. In addition, structured lipids, a type of liquid lipid commonly used in parenteral nutrition, were selected to construct NE with lecithin for its good solubility of LBT-OA rather than other liquid lipids (eg, soybean oil and medium-chain triglyceride). The physicochemical properties and in vivo pharmacokinetics of both LBT-OA-NE and LBT-OA-PEG-NE were further evaluated and compared with free LBT. The biodistribution of these NE formulations was examined to estimate their targeting performance in rats. Afterward, the PEGylated NE and free LBT were compared with respect to heterotopic and lung metastatic tumor models, respectively, to evaluate the antitumor effect.

\section{Materials and methods Materials}

LBT acetate was offered by Sichuan Shifang Hongsheng Plant Raw Material Co, Ltd (Shifang, People's Republic of China). Purified yolk lecithin (Lipoid E80), mPEG2000-DSPE, and OA were purchased from Lipoid Co, Ltd (Ludwigshafen, Germany). The structured triglyceride (structured lipid [SL]) was a gift from Sichuan Baili Pharmaceutical Group Co, Ltd (Chengdu, Sichuan, People's Republic of China). All the other chemical solvents and reagents were of analytical grade or above.

\section{Animals}

Male Wistar rats $(200 \pm 20 \mathrm{~g})$ and eight-week-old C57BL/6 mice were obtained from the Laboratory Animal Center of Sichuan University (Chengdu, People's Republic of China). They were fed in sterile conditions and acclimated for at least 5 days. All the animal experiment protocols and procedures were approved and supervised by the Animal Ethics Committee at Sichuan University.

\section{Preparation of LBT-OA}

To neutralize the charges of cationic LBT acetate salt form and facilitate drug entrapment in the oil phase, LBT-OA was prepared using a coprecipitation method. The amount of LBT used was calculated as the acetate salt form throughout the experiment. To prepare an aqueous solution of LBT acetate $(1.6 \mathrm{mg} / \mathrm{mL})$, 
$20 \mu \mathrm{L}$ of $5 \%$ acetic acid solution was added to $8 \mathrm{~mL}$ of aqueous solution of LBT acetate $(1.6 \mathrm{mg} / \mathrm{mL})$ in a $10 \mathrm{~mL}$ centrifuge tube. An aqueous solution of LBT base was prepared by adding $0.14 \mathrm{~mL}$ of sodium bicarbonate solution $(50 \mathrm{mg} / \mathrm{mL})$ to the above solution. LBT-OA was prepared by adding $0.25 \mathrm{~mL}$ of ethanol solution of OA $(100 \mathrm{mg} / \mathrm{mL})$ to the obtained LBT-base solution. Under continuous vortex mixing at room temperature, a cloudy solution spontaneously formed as a result of the complex formation. LBT-OA, a yellow precipitate, was collected via centrifugation. The supernatant was diluted with $1 \%$ acetic acid ethanol solution $(1 \mathrm{~mL}$ of acetic acid in $100 \mathrm{~mL}$ of ethanol) and subsequently quantified (excitation/emission: $367 / 450 \mathrm{~nm}$ ) by fluorescence spectrophotometer (RF-5301 PC, Shimadzu Scientific Instruments, Kyoto, Japan) to determine the concentration of LBT in the supernatant. The percentage of LBT complexed with OA was calculated as follows:

Percentage of LBT ion-paired with OA

$=[1-($ Drug's weight in supernatant $/$ The total weight of the added drug)] $\times 100 \%$.

The resultant complex was washed three times with water for injection, sealed, and kept in a desiccator at room temperature.

\section{Determination of octanol/water partition coefficient}

The octanol/water partition experiment was performed by the shaking-flask method using the following experimental conditions. The LBT base was prepared by the addition of excess sodium bicarbonate to an aqueous solution of LBT acetate, followed by an extract ion into a dichloromethane and solvent evaporation. Water and octanol were mutually saturated for 24 hours before the experiment. First, $1 \mathrm{~mL}$ of aqueous LBT acetate $(1 \mathrm{mg} / \mathrm{mL})$ and $1 \mathrm{~mL}$ of aqueous LBT base $(1 \mathrm{mg} / \mathrm{mL})$ were mixed with $3 \mathrm{~mL}$ of octanol, respectively. The $1 \mathrm{~mL}$ octanol solution of LBT-OA complex (equivalent to $1 \mathrm{mg} / \mathrm{mL}$ LBT base) was mixed with $3 \mathrm{~mL}$ of water. Consequently, the two phases were vigorously vortexed for 10 minutes and agitated for 24 hours in a thermostatic shaker bath (TaiCang Digitek Instrument Co, Ltd, TaiCang, JiangSu Province, People's Republic of China) at $25^{\circ} \mathrm{C} \pm 0.1^{\circ} \mathrm{C}$. After equilibration, samples were centrifuged at $18,000 \times \mathrm{g}$ at $25^{\circ} \mathrm{C}$ for 15 minutes. Then, both phases were diluted with $1 \%$ acetic acid in ethanol and subsequently assayed by fluorescence spectrophotometry to determine the concentration. Each experiment was performed at least in triplicate.
The partition coefficient $\left(\log \mathrm{P}_{\mathrm{o} / \mathrm{w}}\right)$ was calculated by the following equation:

$$
\log \mathrm{P}_{\mathrm{o} / \mathrm{w}}=\log \left[\left(\mathrm{C}_{\mathrm{o}} \times \mathrm{V}_{\mathrm{o}}\right) /\left(\mathrm{C}_{\mathrm{w}} \times \mathrm{V}_{\mathrm{w}}\right)\right]
$$

$\mathrm{C}_{\mathrm{o}}$ : concentration of LBT in octanol at equilibrium; $\mathrm{V}_{\mathrm{o}}$ : volume of octanol in sample; $\mathrm{C}_{\mathrm{w}}$ : concentration of LBT in water at equilibrium; $\mathrm{V}_{\mathrm{w}}$ : volume of water in sample.

\section{Differential scanning calorimetry measurement}

Samples of LBT, OA, LBT-OA, and a mixture of LBT and OA were placed in vacuum at ambient temperature for two days to remove solvent residuals completely. Samples were then hermetically sealed in an aluminum pan and heated at a rate of $10^{\circ} \mathrm{C}$ per minute from $28^{\circ} \mathrm{C}$ to $300^{\circ} \mathrm{C}$. The melting energy of the crystalline part of the polymer was obtained from the area of the endothermic peak.

\section{Preparation of LBT-OA loaded NE and blank NE}

LBT-OA-NE was prepared by a simple lipid film hydrationhigh pressure homogenization method. Briefly, LBT-OA prepared from $100 \mathrm{mg}$ LBT and $250 \mathrm{mg}$ OA, $800 \mathrm{mg}$ lipoid E80, and $500 \mu \mathrm{L}$ structured triglyceride was dissolved in $200 \mathrm{~mL}$ of dichloromethane in a round bottom flask. The organic phase was subsequently removed by a R-144 rotary evaporator (Buchi Laboratory Equipment, Flawil, Switzerland) under reduced pressure at $45^{\circ} \mathrm{C}$ until a thin lipid film formed. The dried lipid film was rehydrated in $50 \mathrm{~mL}$ water, followed by vigorous vortex. Afterward, this predispersion was passed through a high-pressure homogenizer (EmulsiFlex-C5, AVESTIN Inc, ON, Canada) for seven cycles at an operating pressure of $96.4 \mathrm{MPa} 115.6 \mathrm{MPa}$, resulting in the formation of LBT-OA-NE solution. LBT-OA-PEG-NE was prepared the same as LBT-OA-NE. The only difference was that $760 \mathrm{mg}$ lipoid E80 and $150 \mathrm{mg}$ mPEG2000-DSPE was added into dichloromethane instead of $800 \mathrm{mg}$ lipoid E80 (Table 1). Blank PEG-NE was prepared the same as LBT-OA-PEGNE just without LBT.

\section{Characterization and fixed aqueous layer thickness of NE}

The NE's particle size and zeta potential were measured by photon correlation spectroscopy (Malvern Zetasizer Nano ZS90, Malvern Instruments, Malvern, UK) in water at $25^{\circ} \mathrm{C}$. Then the morphology of NE was observed by a transmission 
Table I Components of each formulation

\begin{tabular}{lll}
\hline Composition & LBT-OA-NE & LBT-OA-PEG-NE \\
\hline LBT $(\mathrm{mg})$ & 100 & 100 \\
OA $(\mathrm{mg})$ & 250 & 250 \\
E80 $(\mathrm{mg})$ & 800 & 760 \\
mPEG2000-DSPE $(\mathrm{mg})$ & 0 & 150 \\
Structured lipid $(\mu \mathrm{L})$ & 500 & 500 \\
\hline
\end{tabular}

Abbreviations: LBT, lycobetaine; NE, nanoemulsion; OA, oleic acid; E80, injectable egg lecithin; PEG, polyethylene glycol; mPEG2000-DSPE, $\mathrm{N}$-(carbonyl-methoxypolyethylene-glycol-2000)-I,2-distearoyl-sn-glycero-3-phospho ethanolamine.

electron microscope instrument (H-600, Hitachi, Tokyo, Japan). Based on the Gouy-Chapman theory, ${ }^{14}$ the fixed aqueous layer thickness of the NE is expressed as:

$$
\ln F[L]=\ln A-k L \text {. }
$$

$F[L]$ is zeta potential (the electrostatic potential at the position of the slipping plane), $A$ is a constant, and $k$ is calculated by $\sqrt{c / 0.3}$ ( $c$ is the electrolyte's molality). The zeta potentials are measured with the increasing concentration of $\mathrm{NaCl}(0,10,50$, and $100 \mathrm{mM})$. After the zeta potential was plotted against $k$, the slope $L(\mathrm{~nm})$ represents the fixed aqueous layer thickness (FALT).

\section{Determination of drug-loading parameters and entrapment efficiency}

The entrapment efficiency of LBT-OA-NE and LBT-OAPEG-NE were determined by the ultrafiltration method. To separate the free drug from the NE suspension, Nanosep ${ }^{\circledR}$ Centrifugal Filtration Devices (Mw cut-off $300 \mathrm{kDa}$; Pall Corporation, Port Washington, NY, USA) were used. A fixed volume $(400 \mu \mathrm{L})$ of the freshly prepared LBT-OA-NE or LBT-OA-PEG-NE $(1 \mathrm{mg} / \mathrm{mL})$ was added to the sample reservoir tube and spun at $14,000 \times \mathrm{g}$ at $25^{\circ} \mathrm{C}$ for 60 minutes. The collected filtrate in the retentate vial was diluted with $1 \%$ acetic acid in ethanol $(1 \mathrm{~mL}$ acetic acid in $100 \mathrm{~mL})$ and analyzed by fluorescence spectrophotometry RF5301 PC (Shimadzu Scientific Instruments, Kyoto, Japan) (excitation wavelength: $367 \mathrm{~nm}$; emission wavelength: $450 \mathrm{~nm}$ ).

The formula of drug-loading (DL) coefficient and encapsulation efficiency (EE) were listed as:

$$
\begin{gathered}
\text { DL } \%=\frac{\text { Weight of the drug added }}{\text { Weight of the drug and excipients added }} \times 100 \% \\
\mathrm{EE} \%=\frac{\text { Weight of the drug in filtrate }}{\text { Weight of the feeding drug }} \times 100 \% .
\end{gathered}
$$

\section{Drug release studies}

Drug release from LBT-OA-NE and LBT-OA-PEG-NE was investigated using a dialysis bag-diffusion technique. phosphate buffer saline (PBS) (100 mL; 0.01 M; pH 7.4) was used as the release medium at $37^{\circ} \mathrm{C} \pm 0.1^{\circ} \mathrm{C}$, under constant shaking at $100 \mathrm{rpm}$ in a thermostated shaker bath (TaiCang Digitek Instrument Co, Ltd, TaiCang, JiangSu Province, People's Republic of China). An appropriate amount of LBT, LBT-OA-NE, and LBT-OA-PEG-NE was suspended in the cellulose-membrane dialysis bag (Mw cut-off: 8000-14,000; EMD Millipore Corporation, Billerica, MA, USA) and immersed into the release medium. At certain time intervals, $2 \mathrm{~mL}$ aliquot of the medium was pipetted out, and each withdrawal was followed by replacement with the same volume of fresh medium. The amount of LBT released in the supernatant was determined by fluorescence spectrophotometry after the sample solution had been diluted with $1 \%$ acetic acid in ethanol RF5301 PC (Shimadzu Scientific Instruments, Kyoto, Japan) (excitation wavelength: $367 \mathrm{~nm}$; emission wavelength: $450 \mathrm{~nm}$ ). A control experiment to determine the release behavior of the free drug was also performed. An appropriate amount of LBT was dissolved in water (adjusted to $\mathrm{pH} 5 \sim 6$ with acetic acid), and $1 \mathrm{~mL}$ of this solution was enclosed in a dialysis bag and was immersed into the release medium, at $37^{\circ} \mathrm{C} \pm 0.1^{\circ} \mathrm{C}$. Then, the procedure described above for the NE was followed. Each experiment was performed in triplicate.

\section{Pharmacokinetic studies in rats}

The LBT-OA-NE, LBT-OA-PEG-NE, and LBT solutions were administered to Wistar rats via the tail vein at a dose of $10 \mathrm{mg} / \mathrm{kg}$ (about $1 \mathrm{~mL}$ per rat). Five rats were used in each group, and blood was collected from the orbit at a predetermined time after the intravenous injection, such as 1 minute, 3 minutes, 6 minutes, 15 minutes, 30 minutes, 1 hour, 2 hours, 4 hours, 6 hours, 8 hours, and 10 hours. Plasma samples were obtained following centrifugation at $5000 \mathrm{rpm}$ for 10 minutes.

Plasma samples $(0.1 \mathrm{~mL})$ were mixed with $20 \mu \mathrm{L}$ of $20 \%$ trichloroacetic acid solution to precipitate protein. The mixtures were vortexed for 5 minutes. Then, they were centrifuged for 10 minutes at $13,500 \mathrm{rpm}$, and $280 \mu \mathrm{L}$ of $1 \%$ acetic acid solution was added to the samples to ion pair with LBT. After the mixtures were vortexed for 5 minutes, they were centrifuged for 10 minutes at 13,500 rpm. Twenty $\mu \mathrm{L}$ of the supernatants were injected into the high-performance liquid chromatography. 


\section{Biodistribution studies in rats}

The LBT-OA-NE, LBT-OA-PEG-NE, and LBT solutions were administrated to Wistar rats via the tail vein at a dose of $10 \mathrm{mg}$ per $\mathrm{kg}$. Five rats were used at each predetermined time after intravenous injection (15 minutes, 1 hour, 4 hours), and their blood and tissues (heart, lung, liver, kidney, and spleen) were collected.

Every tissue sample was accurately weighed, homogenized, and was given two volumes of $0.9 \%$ $\mathrm{NaCl}$ solution to gain a concentration of $333.33 \mathrm{mg} / \mathrm{mL}$. Homogenized tissue samples (heart, spleen, liver, lung, and kidney; $0.1 \mathrm{~mL})$ and plasma samples $(0.1 \mathrm{~mL})$ were mixed with $20 \mu \mathrm{L} 20 \%$ trichloroacetic acid solution to precipitate protein. After the mixtures were vortexed for 5 minutes, they were centrifuged at $13,500 \mathrm{rpm}$ for 10 minutes. Then, a $280 \mu \mathrm{L} 1 \%$ acetic acid solution was added to the samples to ionize with the LBT. After the mixtures were vortexed for 5 minutes, they were centrifuged at $13,500 \mathrm{rpm}$ for 10 minutes. Then, $20 \mu \mathrm{L}$ of the clear supernatants was injected into the high-performance liquid chromatography (HPLC) system.

\section{HPLC analysis}

The HPLC system was Agilent 1260 (USA) and Kromasil $\mathrm{C}_{18}$ column $(150 \mathrm{~mm} \times 4.6 \mathrm{~mm} ; 5 \mu \mathrm{m})$. The mobile phase was a mixture of $10 \mathrm{mmol} / \mathrm{mL}$ ammonium acetate (1\% triethylamine; adjusted to $\mathrm{pH} 3.6$ with acetic acid) and methanol (74:26; volume/volume). The measurement wavelength was $260 \mathrm{~nm}$; the measurement temperature was $35^{\circ} \mathrm{C}$, and the flow rate was $1.0 \mathrm{~mL}$ per minute.

\section{Pharmacokinetics data analysis}

The data was analyzed using nonlinear regression analysis by the computer program Drug and Statistics Software 2.0 (Drug and Statistics, Anhui, People's Republic of China).

\section{Cell culture}

The C57BL/6-derived Lewis lung carcinoma cell line and the B16F10 cell line (the lung high metastasis cell lines, B16F10) were purchased from the American Type Culture Collection (Manassas, VA, USA). Both cells were cultured in RPMI-1640 medium (GIBCO Products International, Langley, OK, USA) supplemented with $10 \%$ fetal bovine serum (Minhai Bio-Engineering Co, Ltd, Gansu, People's Republic of China), penicillin $(100 \mathrm{U} / \mathrm{mL})$ and streptomycin $(0.1 \mathrm{mg} / \mathrm{mL})$ at $37^{\circ} \mathrm{C}$ in humidified atmosphere containing $5 \% \mathrm{CO}_{2}$ in air.

\section{Antitumor activity in heterotopic models}

The heterotopic Lewis lung carcinoma (LLC) tumor model was obtained by the subcutaneous injection of LLC cells $\left(3 \times 10^{6}\right.$ cells per $\left.100 \mu \mathrm{L}\right)$ into mice. The model was obtained when the tumors reached about $0.5 \mathrm{~cm}$ in diameter.

To evaluate the antitumor activity in the heterotopic model, four groups (eight mice in each group) were obtained on day 10 after a subcutaneous injection of $100 \mu \mathrm{L}$ LLC cells. The four groups were treated by LBT-OAPEG-NE, LBT solution, the same volume of blank NE, and the same volume of saline solution, three times in 3 days from tail vein, respectively. The inhibitory effect was evaluated by the volume of the heterotopic tumor and the survival rate after treatment. Tumor volume was calculated as following:

Tumor volume $\left(\mathrm{mm}^{3}\right)=0.5 \times$ Width $^{2} \times$ Length

The life-prolonging effect was evaluated by the survival time (days after treatment). Moreover, during treatment, possible side effects the animals had were also observed. If the mice suffered severely (the tumor's diameter exceeded $25 \mathrm{~mm}$ ), they were euthanized. Survival curves were established using the Statistical Product and Service Solutions 17.0 (SPSS Inc., Chicago, IL, USA).

\section{Antitumor activity in lung metastasis model}

The lung metastasis model was obtained by tail vein injection of B16F10 cells $\left(3 \times 10^{5}\right.$ cells per $\left.100 \mu \mathrm{L}\right)$ into mice. The experiments were started when the tumors were successfully inoculated into the lung (about 23 days after inoculation).

To evaluate the antitumor activity of LBT-OA-PEG-NE in the lung metastasis model, four groups (ten mice in each group) were obtained on day 23 after tail vein injection of $\mathrm{B} 16 \mathrm{~F} 10$. The four groups were given LBT-OA-PEG-NE, LBT solution, the same volume of blank NE and the same volume of saline solution three times in three days from the tail vein, respectively. The antitumor effect and life-prolonging effect were evaluated by the survival rate. Moreover, during treatment, possible side effects of animals were also observed. If the mice suffered severely, they were euthanized. Survival curves were established using the Statistical Package for the Social Sciences 17.0 (IBM Corporation, Armonk, NY, USA). 


\section{Statistical analysis}

All the experiments were repeated at least three times. All values are expressed as mean value plus or minus standard deviation. The statistical analysis of the samples was using a Statistical Package for the Social Sciences 17.0 (IBM Corporation) $t$-test with $P<0.05$ as the minimal level of significance.

\section{Results and discussion Preparation of LBT-OA}

Ion-pair formation is a method for modifying the lipophilicity of ionizable drugs that has been used successfully by shielding their charge with an oppositely charged ion without chemical modifications. ${ }^{15,16}$ LBT has the quaternary ammonium moieties, which bear positive electrostatic charges localized at the protonated amino nitrogen. LBT could thus ionize with $\mathrm{OA}$. The resulting ion-pair complexes increased the lipophilicity of LBT and facilitated the incorporation of LBT into the NE. Anionic ion-pairing agents, sodium taurodeoxycholate, and sodium tetradecyl sulfate, had been used to neutralize the charges of the cationic drug and enhance the entrapment of the drug in the nanoparticles. ${ }^{17}$ However, the major disadvantage of the ion-pair method is that the ion-pairing agents are potentially toxic and cannot be utilized for intravenous injection. In a former study, ${ }^{18} \mathrm{OA}$ was used as the complexing agent, because $\mathrm{OA}$ is biodegradable and the physiological long chain fatty acid shows low toxicity. In addition, it is being used for parenteral formulations clinically. ${ }^{19}$ Therefore, OA was selected in this study to ion-pair with LBT. Due to LBT's instability, it decomposes easily in a strong alkaline environment. Sodium bicarbonate solution was selected to change the $\mathrm{pH}$ value instead of other bases, such as sodium hydroxide. When increasing the $\mathrm{pH}$, it is probable that a deprotonation of the phenolic group occurred at basic $\mathrm{pH}$, thus neutralizing the positive charge of the molecule and consequently rendering the molecule more lipohilic. In the preparation process, OA was dissolved in ethanol, and the solution was added to the aqueous solution immediately. When the OA was added to the sodium bicarbonate solution, it is probable that the deprotonation of OA to sodium oleate occurs, and then sodium oleate interacts with LBT ammonium quaternary salt to form the ion pair (Figure 1). In this way, OA is dispersed in an aqueous solution more easily than adding OA directly into the LBT solution and, as a result, it could react with the LBT sufficiently. The codissolve-evaporation method can be used to prepare LBT-OA. ${ }^{20,21}$
Briefly, LBT-OA was prepared by dissolving $10 \mathrm{mg}$ of LBT base and $25 \mathrm{mg}$ of OA in dichloromethane, followed by solvent evaporation at $30^{\circ} \mathrm{C}$ under reduced pressure. Compared with the codissolve-evaporation method, the stirring method avoided using a toxic organic solvent, and the process was more convenient. The formation of an ionic complex is generally driven by electrostatic attraction, hydrophobic interaction, and hydrogen bonding. ${ }^{16}$ Although solvents with a low dielectric constant were suitable for ionpair formation, once the ion pair is formed in an aqueous solution, the hydrophobic interaction also can successfully form ion pair as well as electrostatic force. ${ }^{22}$ This explained why the LBT-OA can be prepared both in water and dichloromethane. Phospholipids has been reported that the ionic complexes obtained by interactions between aliphatic amines and carboxylic acids have a structure type of the ion pair and complex composition of $1: 1 .^{23}$ Therefore, LBT-OA was hypothesized to form through electrostatic forces derived from the charge neutralization between the quaternary ammonium group of LBT and the acidic carboxyl group of OA (Figure 1). However, the quaternary ammonium group in LBT was too weak to react with the OA completely. Thus, the amount of OA was increased to almost three times the amount of LBT (2.88:1, $\mathrm{mol} / \mathrm{mol})$ to ensure the formation of LBT-OA. The extra amount of OA was also an effective counter-ion for LBT in the NE formulation. The addition of OA to aqueous solution of LBT base resulted in the immediate formation of ion-pair complex as brownish precipitates. During this complexation process, more than $95.1 \%$ of the LBT could ion-pair with OA, which was almost complete. Results also showed the formed LBT-OA was highly soluble in organic solvents, especially in ethanol, ethyl acetate, and acetone (data not shown).

\section{Determination of octanol/water partition coefficient}

The octanol/water partition coefficient $\left(\log \mathrm{P}_{\mathrm{O} / \mathrm{W}}\right)$ of LBT acetate, LBT base and LBT-OA was $-2.41,0.36$, and 1.12 , respectively. It was shown that the lipophilicity of LBT base was slightly stronger than that of the LBT acetate. Moreover, the lipophilicity of LBT-OA was much stronger than that of the LBT acetate. These results indicated that the ion pair that formed between LBT and OA could shield the positive charge of LBT and that the long alkyl chain of OA increased the lipophilicity of LBT$\mathrm{OA}$, thereby facilitating the distribution of LBT in the organic phase. The enhanced lipophilicity of LBT-OA contributed to the increased solubility of LBT in the lipid matrix and, therefore, improved the entrapment efficacy of LBT in NE. 


\section{Differential scanning calorimetry}

The differential scanning calorimetry curves of LBT acetate, OA, LBT and OA physical mixtures, and LBT-OA complex are shown in Figure 2. OA showed a broad endothermal peak at $283.0^{\circ} \mathrm{C}$, corresponding to the evaporating behavior of OA. LBT acetate displayed a sharp-point endothermal peak at $226.1^{\circ} \mathrm{C}$, corresponding to its melting point. The physical mixture of LBT and OA showed two endothermal peaks. One was at $45.6^{\circ} \mathrm{C}$, similar to the onset temperature of OA complex, and the other was around $286.4^{\circ} \mathrm{C}$, similar to the onset temperature of OA. The first peak of physical mixture could be interpreted as when the temperature was increased, OA melted, and drugs were dissolved in the OA and partly formed OA complex, which could be explained through the theory of preparation by the melt-out method. The differential scanning calorimetry curves of OA complexes showed the disappearance of endothermal peaks of drug and $\mathrm{OA}$, indicating the possible interactions between LBT and $\mathrm{OA}$, such as the formation of hydrogen bonds or van der Waals forces, rendering the loss of the second endothermal peak of OA.

\section{Preparation of LBT-OA-NE and LBT-OA-PEG-NE}

In this study, we developed an economical, simple and reproducible method. High pressure homogenization (HPH) was proven a reliable technique for the preparation of NE that is being used in industry. In our study, NE consisted of lecithin, $\mathrm{OA}$, and structured lipids (SL) (injectable grade) (Table 1). All ingredients were biodegradable and physiological lipids showed low toxicity. ${ }^{24}$ Moreover, these materials are accepted by the regulatory authorities, such as the US Food and Drug Administration (FDA), and are clinically available for intravenous injection. In most cases, surfactants are added to the formulation of NE to stabilize fine particles with increasing surface area by preventing the coalescence of droplets. However, many of these stabilizers showed low biocompatibility in parenteral administration. In the formulation of LBT-OA-NE and LBT-OA-PEG-NE, the only surfactant used was lecithin (E80 and mPEG2000-DSPE), which was native and well-tolerated in humans. ${ }^{25}$ Moreover, it has been widely used in parenteral nutrition emulsions. Therefore, the two formulations developed in this study should expect fewer concerns with surfactant toxicity. The drug/lipid ratio and the amount of SL were only optimized in the formulation of LBT-OA-NE. However, mPEG2000-DSPE was added in the formulation of LBT-OA-PEG-NE instead of part of E80. Therefore, different process variables were employed to optimize the LBT-OA-NE preparation. The influences of LBT/phospholipid ratio (weight/weight [w/w]), LBT/SL ratio $(\mathrm{w} / \mathrm{w})$, and the conditions of $\mathrm{HPH}$ on the particle size distribution and the polydispersity index were examined. The size and morphology of NE were significantly impacted by the amount of phospholipids. Phospholipids could successfully form NE without micron-grade particles when the ratio of LBT/phospholipid reached 1:8 or 1:10 (Table 2). For the intravenous injection purposes, fewer excipients would be better. Therefore, we employed LBT/phospholipid at the ratio of 1:8 to prepare NE, resulting in a relatively optimal particle size and polydispersity. The phospholipid or the excess OA contributes to the negative charges of $\mathrm{NE},{ }^{26}$ making them dynamically stable. In this system, higher drug-

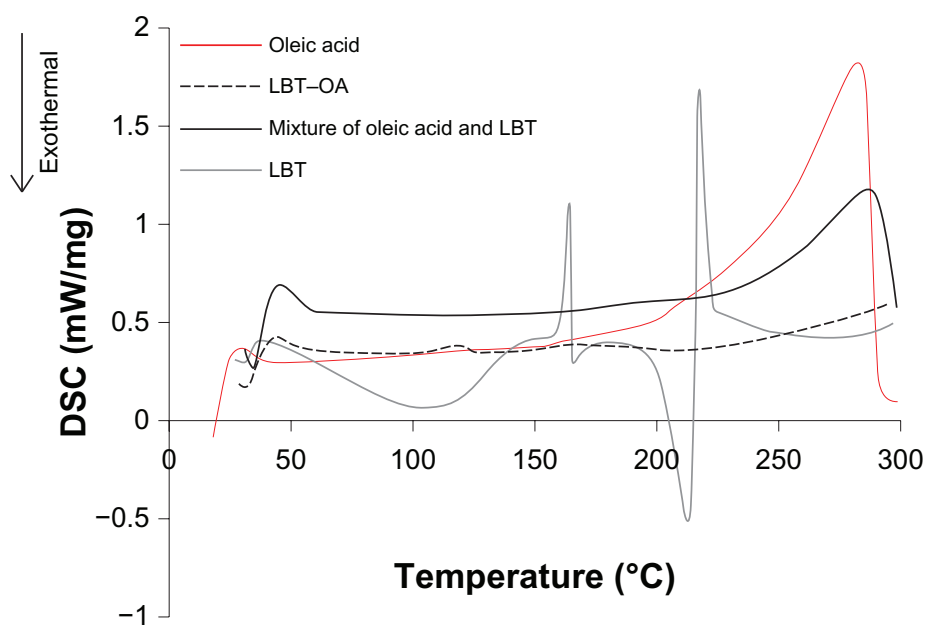

Figure 2 DSC thermograms of oleic acid, lycobetaine acetate, lycobetaine-oleic acid complex (LBT-OA) and physical mixture of lycobetaine acetate and oleic acid. Abbreviations: LBT, lycobetaine; OA, oleic acid; DSC, differential scanning calorimetry. 
Table 2 Formulations screening of LBT-OA-NE

\begin{tabular}{llllll}
\hline LBT $(\mathbf{m g})$ & E80 $(\mathbf{m g})$ & $\begin{array}{l}\text { Structured } \\
\text { lipids }(\mathbf{m g})\end{array}$ & $\begin{array}{l}\text { Z-average } \\
\text { diameter }(\mathbf{n m})\end{array}$ & $\begin{array}{l}\text { Polydispersity } \\
\text { index }\end{array}$ & $\begin{array}{l}\text { Micron-grade } \\
\text { large particles }\end{array}$ \\
\hline 100 & 200 & 50 & $248.0 \pm 9.9$ & $0.271 \pm 0.012$ & + \\
100 & 400 & 50 & $204.8 \pm 5.8$ & $0.236 \pm 0.015$ & + \\
100 & 600 & 50 & $205.8 \pm 4.9$ & $0.234 \pm 0.008$ & + \\
100 & 800 & 50 & $170.6 \pm 4.8$ & $0.155 \pm 0.009$ & - \\
100 & 1000 & 50 & $170.8 \pm 3.8$ & $0.204 \pm 0.010$ & - \\
100 & 800 & 20 & $169.8 \pm 3.2$ & $0.226 \pm 0.008$ & + \\
100 & 800 & 40 & $198.0 \pm 2.0$ & $0.194 \pm 0.005$ & - \\
100 & 800 & 50 & $170.6 \pm 4.8$ & $0.155 \pm 0.009$ & - \\
100 & 800 & 60 & $178.5 \pm 0.6$ & $0.195 \pm 0.007$ & - \\
100 & 800 & 80 & $177.7 \pm 1.4$ & $0.170 \pm 0.010$ & - \\
\hline
\end{tabular}

Notes: "+" means there are micron grade large particles in the NE; and "-"means not.

Abbreviations: LBT, lycobetaine; NE, nanoemulsion; OA, oleic acid; E80, injectable egg lecithin.

loading efficiency was achieved by LBT-OA-NE, avoiding the administration of redundant excipients.

Due to the increased liposolubility of LBT-OA, only a small amount of SL lipids (LBT/SL; 1:5; w/v) was required to prepare the NE with an optimal size distribution and polydispersity index. As shown in Table 2, when the LBT/ phospholipid/SL ratio (w/w/v) reached 1:8:5, both the mean size and polydispersity index (PDI) reached the best result. To study the conditions of HPH, the influence parameters, such as applied pressure, homogenization cycles, and temperature on particle-size distribution, were investigated. The temperature affected the size distribution of LBT-OA-NE slightly, due to the fact that the melting point of the lipids used in LBT-OA-NE was relatively low, so the homogenization process could be performed at room temperature to simplify the preparation. Studies showed seven homogenization cycles at an operating pressure of $100 \mathrm{MPa}$ were sufficient to prepare LBT-OA-NE with optimal particle size and PDI. Increasing the homogenization pressure or the number of cycles resulted in an increase in the particle size, due to the droplets' coalescence which occurred as a result of the high kinetic energy of the NE droplets. In this experiment, the amount of mPEG2000-DSPE was $5 \mathrm{~mol} \%$ of E80. In every formulation of this experiment, the molar ratio of E80 and mPEG2000-DSPE was fixed as following:

mPEG2000-DSPE: E80 $=x:(1-x)(x$ was $0.05,0.1$, or 0.2$)$

Increasing the amount to $10 \mathrm{~mol} \%$ or $20 \mathrm{~mol} \%$ of E 80 , the result showed extraordinarily small particle size, less than $100 \mathrm{~nm}$, with micelle-like morphology rather than NE. On the other hand, an excess of PEGylated lecithin ( $>10$ mol\%) may cause the "accelerated blood clearance phenomenon," which makes PEGylated nanoparticles lose their sustained circulation characteristics via repeated injection. ${ }^{27}$

\section{Characteristics of NE}

NE with and without $5 \mathrm{~mol} \% \mathrm{mPEG} 2000$-DSPE were prepared. The NE properties are showed in Table 3. The size distribution of NE measured by photon correlation spectroscopy showed one narrow peak, indicating that the NE droplets population was relatively homogenous in size. The calculated mean size of LBT-OA-NE and LBTOA-PEG-NE, based on three separate measurements, was $170.6 \pm 4.8 \mathrm{~nm}$ with PDI of $0.155 \pm 0.009$ and $146.9 \pm 4.3 \mathrm{~nm}$ with a PDI of $0.184 \pm 0.012$, respectively, which indicated that both NE formulations were uniform and monodispersed.

Furthermore, Sriwongsitanont's research showed that the decrease in vesicle size with an increase in $\mathrm{mPEG} 2000$-DSPE was ascribed to the steric hindrance of strongly hydrated PEG. ${ }^{28}$ The mean particle size and a PDI of PEGylated NE were much desirable, due to the optimal formulation and preparation parameters of NE. The transmission electron microscope micrographs of LBT-OA-NE and LBT-OA-

Table 3 Physicochemical parameters of lipid nanoparticles (nanoemulsions)

\begin{tabular}{lll}
\hline Physicochemical & LBT-OA-NE & LBT-OA-PEG-NE \\
\hline Average diameter $(\mathrm{nm})$ & $170.6 \pm 4.8$ & $146.9 \pm 4.3$ \\
Zeta potential $(\mathrm{mV})$ & $-37.4 \pm 2.4$ & $-29.6 \pm 3.9$ \\
PDI & $0.155 \pm 0.009$ & $0.184 \pm 0.012$ \\
EE\% & $95.42 \pm 1.58$ & $97.32 \pm 2.09$ \\
DL\% & 6.12 & 6.09 \\
FALT $(\mathrm{nm})$ & 1.1 & 2.05 \\
\hline
\end{tabular}

Abbreviations: NE, nanoemulsion; PDI, polydispersity index; $\mathrm{EE}$, encapsulation efficiency; DL, drug-loading coefficient; FALT, fixed aqueous layer thickness; LBT, lycobetaine; LBT-OA-NE, nanoemulsion loaded with lycobetaine-oleic acid complex; LBT-OA-PEG-NE, PEGylated LBT-OA-NE. 
PEG-NE showed that the shape of NE was spherical, and the particle size ranged from 100 to $200 \mathrm{~nm}$, corresponding to the photon correlation spectroscopy results (Figure 3 ). The zeta potential of normal NE and the PEGylated NE was $-37.4 \pm 2.4 \mathrm{mV}$ and $-29.6 \pm 3.9 \mathrm{mV}$, respectively, in approximation to the absolute value of $30 \mathrm{mV}$ to satisfy the stability requirement. Zeta potential is essential to the storage stability of colloidal dispersion, small amounts of phosphatidylserine and phosphatidylglycerol (2\%-5\%) in Lipoid E80 resulted in a negative surface charge. ${ }^{26}$ Moreover, due to an accumulation of the negatively charged ionized carboxyl groups at the interface, OA could also act as coemulsifier, leading to more negative zeta potential, which resulted in a higher resistance to coalescence of the droplets. ${ }^{18}$ According to Gouy-Chapman theory, zeta potential is in inverse proportion to the FALT. ${ }^{14}$ Thus, the zeta potential of PEGylated NE, which had thicker FALT, due to the long PEG chain, was smaller than that of those normal NE.

\section{FALT of the NE}

LBT-OA-PEG-NE's surface negative charge was less than normal NE (Table 3). Based on Gouy-Chapman theory, this phenomenon might be caused by the PEG-aqueous layer. The surface PEG chains' hydrodynamic drag prevented the hydrodynamic plane of shear from closing the charge-bearing plane. Therefore, it caused the electrophoretic mobilities to decrease. ${ }^{14}$ As we all know, the negative zeta potential would increase the risk of lipid NE recognition by macrophages. So the PEGylated NE, which had a less-negative zeta potential, was prepared in our experiment. If there were PEGylated, the absolute value of zeta potential of NE decreased more steeply with $\mathrm{NaCl}$ concentration increasing. The FALT of

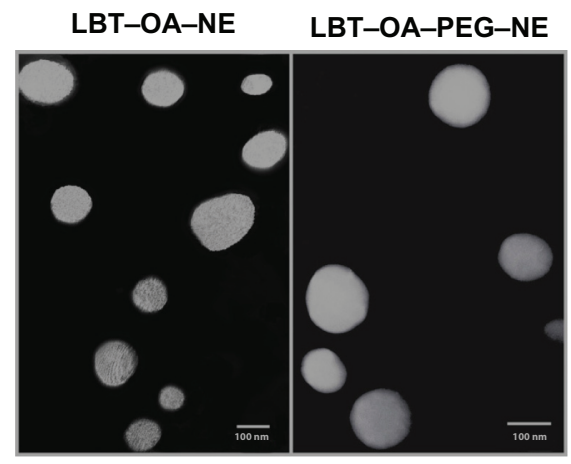

Figure 3 Transmission electron microscopy micrograph of LBT-OA-NE and LBTOA-PEG-NE aqueous suspensions.

Note: Scale bar: $100 \mathrm{~nm}$.

Abbreviations: LBT, lycobetaine; LBT-OA-NE, nanoemulsion loaded with lycobetaine-oleic acid complex; LBT-OA-PEG-NE, PEGylated LBT-OA-NE; $\mathrm{NE}$, nanoemulsion.
LBT-OA-PEG-NE was calculated and shown in Table 1. To prevent flocculation, it should be in a concentration of $\mathrm{NaCl}$ no more than $100 \mathrm{mM}$. The PEGylation would structure a fixed aqueous layer, which may help avoid the adsorption of opsonins and serum proteins, thus allow the escape of NE from the mononuclear phagocyte system. ${ }^{29}$

\section{Drug release studies}

The drug release from the free LBT solution was much faster than from other formulations (Figure 4). The free LBT solution released $46.2 \%$ of LBT within 1 hour. In contrast, the cumulative release amount of LBT-OA-PEG-NE was only $18.9 \%$ within 1 hour, demonstrating that there was no burst effect for LBT-OA-PEG-NE. This phenomenon can be attributed to the increased hydrophobicity of LBT in the formation of LBT-OA. Secondly, a hydrophobic interaction between drug and matrix lipids may help retain the drug in the matrix. Moreover, PEG chains also protected NE from burst effect. More than $80 \%$ of the drug was released from the free LBT solution into the medium after 24 hours, whereas the cumulative amount of LBT released from LBT-OAPEG-NE was less than $55 \%$ by 24 hours, indicating the slow dissolution of LBT from the lipid matrix. The drug release profile of LBT-OA-NE and LBT-OA-PEG-NE could be best characterized by the Korsmeyer-Peppas model and the following regression equation was given in Table 4 obtained by DDsolver 1.0 (China Pharmaceutical University, Nanjing, People's Republic of China), ${ }^{30,31}$ where F $(t)$ is the accumulative drug release $(\%)$, and $t$ is time $(\mathrm{h})$. The release profile of LBT-OA-PEG-NE was biphasic. The initial fast release was observed in the beginning of 8 hours, due to the large surface area of the lipid nanoparticles (LNs) and drug enrich-

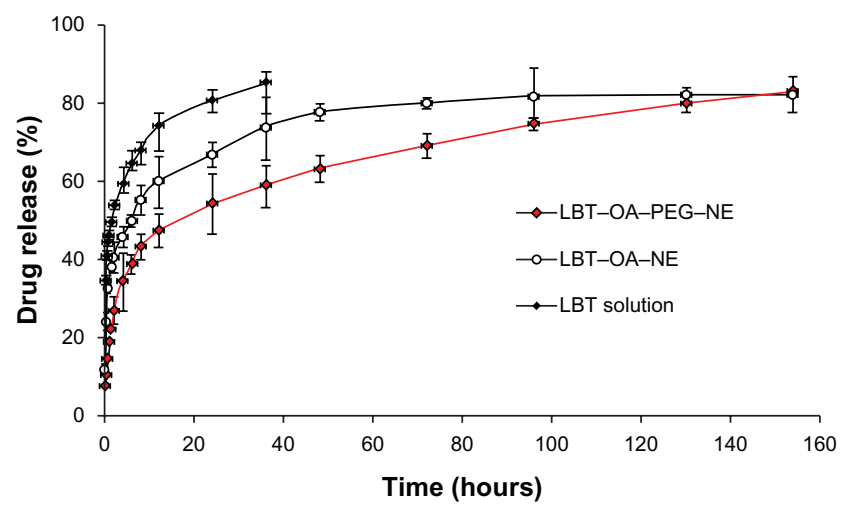

Figure 4 Release curves of LBT from LBT solution, LBT-OA-NE, and LBT-OAPEG-NE in PBS (0.0I M, pH 7.4) $(n=3)$.

Abbreviations: LBT, lycobetaine; LBT-OA-NE, nanoemulsion loaded with lycobetaine-oleic acid complex; LBT-OA-PEG-NE, PEGylated LBT-OA-NE; NE, nanoemulsion. 
Table 4 Release ratio of LBT from LBT-OA-NE and LBT-OAPEG-NE against time by Korsmeyer-Peppas model

\begin{tabular}{lll}
\hline Nanoemulsions & Korsmeyer-Peppas model & $\boldsymbol{R}^{\mathbf{2}}$ \\
\hline LBT-OA-NE & $\mathrm{F}(\mathrm{t})=120.798 \mathrm{t}^{0.077}-86.632$ & 0.9954 \\
LBT-OA-PEG-NE & $\mathrm{F}(\mathrm{t})=51.193 \mathrm{t}^{0.158}-30.487$ & 0.9942 \\
\hline
\end{tabular}

Abbreviations: LBT, lycobetaine; LBT-OA-NE, nanoemulsion loaded with lycobetaine-oleic acid complex; LBT-OA-PEG-NE, PEGylated LBT-OA-NE; NE, nanoemulsion.

ment in the outer shell of the particles. In the late stage, the drug release was constant and slow. It could be attributed to the assumption that the lipophilic LBT-OA solubilized or dispersed in lipid matrix and, therefore, LBT released mainly by dissolution and diffusion of drug from the lipid matrix. In short, the drug release from LBT-OA-PEG-NE was delayed drastically as a result of the increasing lipophilicity of the complex and the incorporation of the drug into the lipid matrix.

\section{Pharmacokinetics of LBT-OA-NE and LBT-OA-PEG-NE in rats}

The blood clearance curves of different LBT formulations are presented in Figure 5. The data was analyzed using nonlinear regression analysis by the computer program Drug and Statistics Software 2.0 (Drug and Statistics, Anhui, People's Republic of China). The area of the concentration-time curve is currently considered to be the standard method to assess the concentration in blood. In pharmacokinetic studies, the area under the concentration-time curve is shown to reflect the circulating situation. The area under the concentration-time curve
(AUC) of LBT of LBT-OA-NE, LBT-OA-PEG-NE in blood after intravenous administration was about 11,32-fold higher than the free LBT. After an injection of LBT-OA-PEG-NE, LBT was present in the plasma of rats after 8 hours. In contrast, the free LBT solution was eliminated quickly and could not be detected in the plasma of those rats after 0.5 hours, and no LBT was detected for LBT-OA-NE at 4 hours after intravenous injection. The release of LBT-OA-NE was slower than that of the LBT solution, it showed the basic ability of NE to slow down the elimination of the drug. The two-compartment model was chosen to fit the concentration-time curves. The pharmacokinetic parameters after intravenous administration of the LBT formulations are reported in Table 5. The plasma kinetics of LBT-OA-PEG-NE showed a higher AUC, a lower rate of clearance and a smaller volume of distribution in comparison to those of the LBT solution and LBT-OA-NE (Table 5). This was probably due to the slower release of LBT from LBT-OA-PEG-NE than from the free LBT solution and even the normal LBT-OA-NE.

This can be attributed to two reasons. First, most of the LBT-OA located in the oil phase of the NE, and some located at the interfacial lecithin layer between the oil phase and the aqueous phase and might release rapidly when the LBT-OA$\mathrm{NE}$ interacted with serum components in the bloodstream. Second, the formulation of LBT-OA-NE did not include the stealth agent, such as the PEGylated lecithin (eg, poly[ethylene glycol]-distearoylphosphatidylethanolamine). Colloidal drug carriers were rapidly removed from systemic circulation after intravenous injection, due to the recognition as foreign bodies by the mononuclear phagocyte system

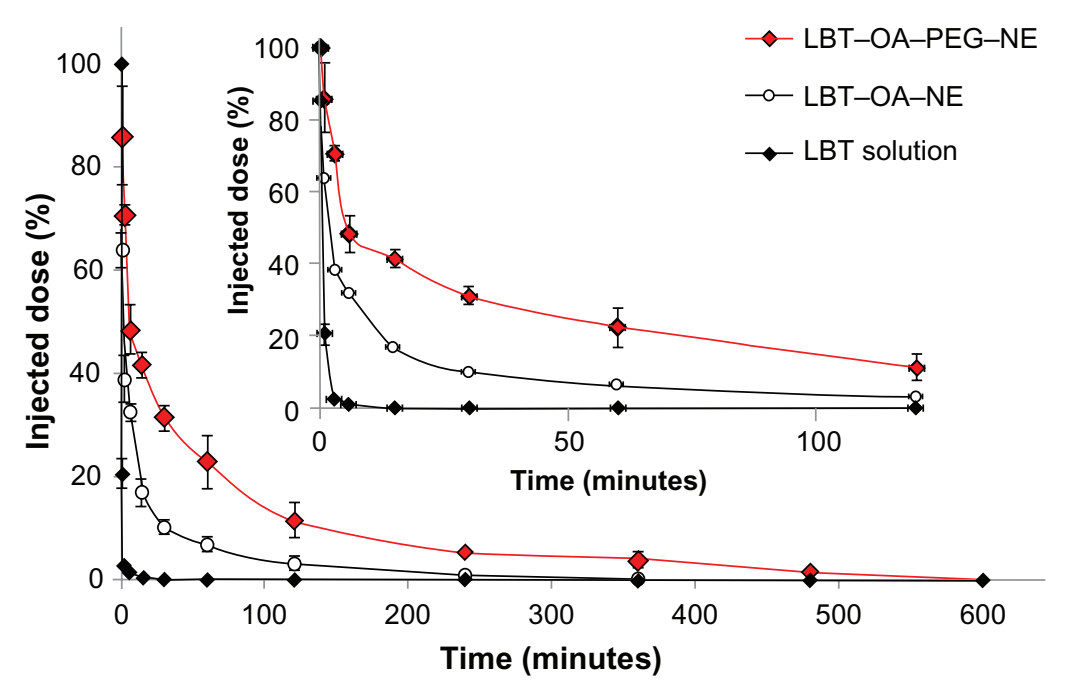

Figure 5 Plasma concentration-time profiles of LBT following intravenous administration of LBT solution, LBT-OA-NE and LBT-OA-PEG-NE (I0 mg/kg). Notes: The inset is detail with enlarged scale from 0 to 100 minutes. Each value represents the mean $\pm S D(n=5)$.

Abbreviations: LBT, lycobetaine; LBT-OA-NE, nanoemulsion loaded with lycobetaine-oleic acid complex; LBT-OA-PEG-NE, PEGylated LBT-OA-NE; NE, nanoemulsion. 
Table 5 Pharmacokinetics parameters of LBT solution, LBT-OA-NE and LBT-OA-PEG-NE in rats after intravenous administration

\begin{tabular}{|c|c|c|c|}
\hline Parameters & $\begin{array}{l}\text { LBT solution } \\
\text { mean (SD) }\end{array}$ & $\begin{array}{l}\text { LBT-OA-NE } \\
\text { mean (SD) }\end{array}$ & $\begin{array}{l}\text { LBT-OA-PEG-NE } \\
\text { mean (SD) }\end{array}$ \\
\hline$t_{1 / 2 \alpha}(\min )$ & $0.424(0.184)$ & $2.018(1.792)$ & $2.856(1.123)^{* * * *}$ \\
\hline$t_{1 / 2 \beta}(\min )$ & $6.951(2.082)$ & $33.447(24.562)^{*}$ & $72.54 \mathrm{I}(13.666)^{* * *, \#}$ \\
\hline $\mathrm{C}_{\max }(\mathrm{mg} / \mathrm{L})$ & 35.965 (4.945) & $52.800(2.730)$ & $57.271(6.373)^{* * * * \#}$ \\
\hline $\mathrm{AUC}_{0-1 \mathrm{Oh}}(\mathrm{mg} / \mathrm{L} \mathrm{min})$ & $112.99(11.68)$ & $1208.16(183.78)^{* * *}$ & $3452.09(458.09) * * * \ldots$ \\
\hline MRT (min) & $2.982(0.369)$ & $50.073(9.666)^{* * *}$ & I I $2.022(7.558)^{* * * \ldots \#}$ \\
\hline $\mathrm{CL}(\mathrm{L} / \mathrm{min} / \mathrm{kg})$ & $0.059(0.007)$ & $0.010(0.003)^{* * *}$ & $0.003(0.001)^{* * *}$ \\
\hline
\end{tabular}

Notes: Data are shown as mean plus or minus standard deviation; $P$-values apply to difference between NE groups and LBT solution; $* P<0.05$;

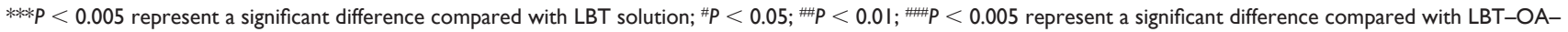
NE; rats were randomly divided into three groups of five.

Abbreviations: $t_{1 / 2 \alpha}$, mean distribution half-life; $t_{1 / 2 \beta}$, mean elimination half-life; AUC ${ }_{0-10 h}$, area under the concentration-time curve from time 0 to I0 hours; MRT, mean residence time; $\mathrm{CL}$, total body clearance; PEG, polyethylene glycol; NE, nanoemulsions; LBT, lycobetaine; LBT-OA-NE, nanoemulsion loaded with lycobetaine-oleic acid complex; LBT-OA-PEG-NE, PEGylated LBT-OA-NE; NE, nanoemulsion.
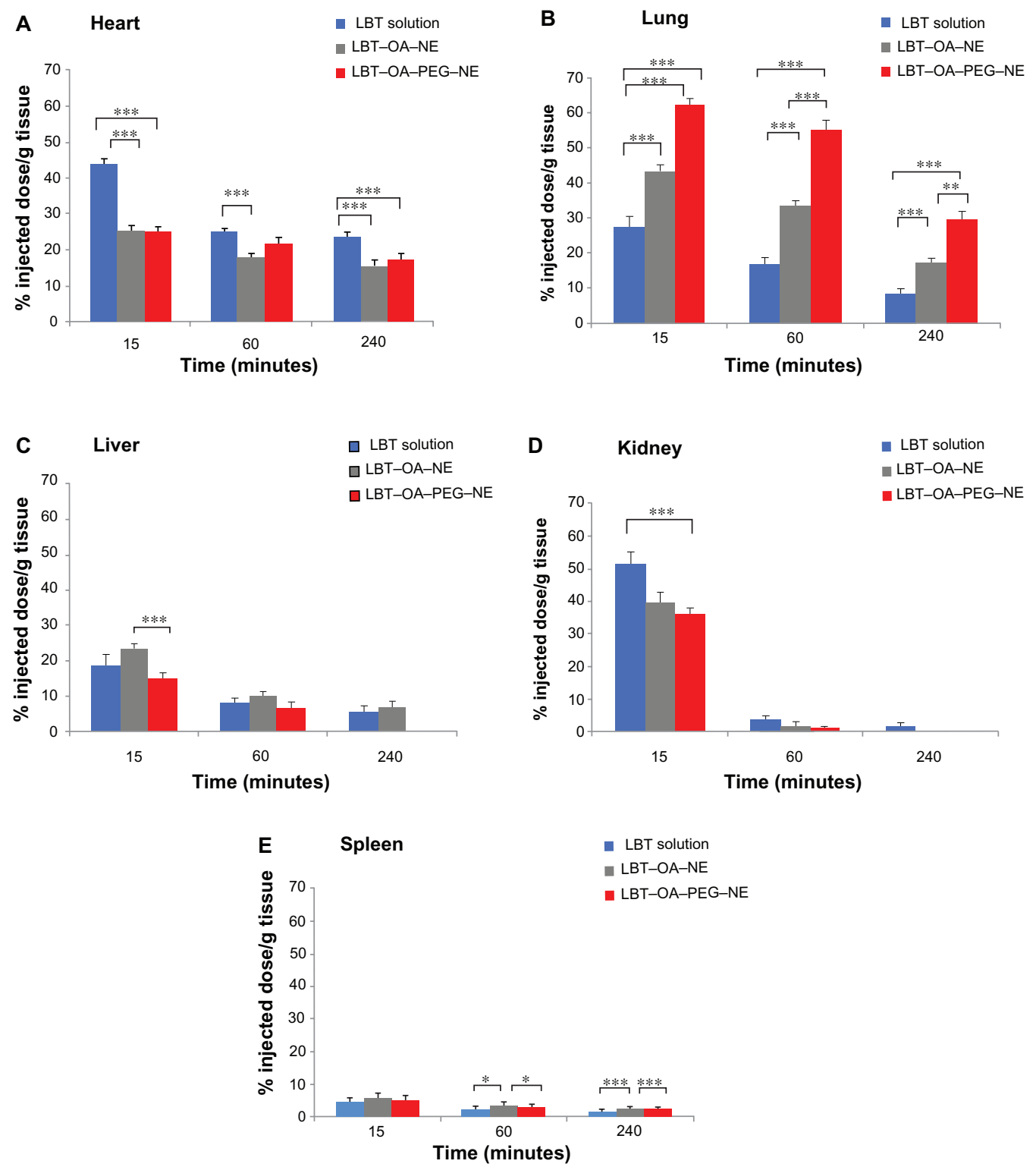

Figure 6 Tissue distribution of LBT after intravenous administration of LBT solution, LBT-OA-NE and LBT-OA-PEG-NE (I0 mg/kg).

Notes: Organ (A-E) accumulation is expressed as percent of injected dose per gram of tissue post-euthanasia at 15 minutes, 60 minutes, and 4 hours. $P$-values apply to difference between nanoemulsions groups and LBT solution; $* P<0.05$; $* * P<0.0$; $* * * P<0.005$ represent a significant difference between two selected groups.

Abbreviations: LBT, lycobetaine; LBT-OA-NE, nanoemulsion loaded with lycobetaine-oleic acid complex; LBT-OA-PEG-NE, PEGylated LBT-OA-NE; NE, nanoemulsion. 
(MPS).$^{32}$ Various parameters, such as the nature of the components, the size, apparent electrical charge, and hydrophilicity of the carriers, influenced the elimination of such colloidal systems. ${ }^{33}$ Coating with hydrophilic polymer chains (PEG and its derivatives) to the surface of the colloidal carriers afforded them a highly hydrophilic shield away from electrostatic and hydrophobic interactions with serum components as well as reduced uptake by MPS. ${ }^{34}$ In comparison to conventional nanocarriers without PEG modification, these PEGylated colloidal drug delivery systems showed a prolonged retention time in blood, primarily due to the reduced recognition and uptake by phagocytic cells of the MPS located mainly in the liver and spleen. Thus, they were less readily cleared from circulation and showed prolonged circulation time in the bloodstream. ${ }^{35,36}$ Therefore, the LBT-OA-PEG-NE protected by the stealth agent was more stable while circulating in the bloodstream than LBT-OA-NE and exhibited relatively longer circulation time. Site-specific delivery of drugs and therapeutics could significantly reduce drug toxicity and increase the therapeutic effect. The pharmacokinetic properties of the drug in the nanocarriers, the vesicle size of the carriers, and the vascular permeability of individual tissues will determine the extravasation and biodistribution profile of the drug-loaded colloidal drug carriers.

\section{Biodistribution of LBT-OA-NE and LBT-OA-PEG-NE in rats}

Biodistribution of LBT in different formulations is shown in Figure 6. In Figure 6B, the LBT's concentration in the lung of LBT-OA-PEG-NE was significantly higher than that of LBT-OA-NE at all times. Moreover, in Figure $6 \mathrm{C}$ and $\mathrm{E}$, the LBT's concentration in the liver of LBT-OA-PEG-NE was significantly lower than that of LBT-OA-NE at 15 minutes, and also in the spleen at 60 minutes and at 240 minutes. So we could infer that LBT-OA-PEG-NE could increase the LBT's concentration in the lung while decreasing it in the liver and spleen. LBT concentration in the lung (a primary target organ for LBT-related antitumor activity) of rats was significantly higher in the LBT-OA-PEG-NE group than in the free LBT solution group after 15 minutes of treatment (Figure 6B). The high concentration of blood could increase the accumulation of LBT in the lung, and the PEG chain could prevent NE uptake by the liver and the spleen. Figure 6 also showed that when LBT-OA-PEG-NE were intravenously administered, LBT in the heart and kidney. This LBT concentration in the heart and kidney at 15 minutes was less than that concentration when treated by LBT solution, respectively. The lower LBT concentration in the heart, liver, and kidney in LBT-OA-PEG-NE-treated rats and the concomitant higher plasma level could be related to a prolonged LBT release from LBT-OA-PEG-NE.

\section{Antitumor activity in lung tumor caused by heterotopic implantation of LLC cells}

After inoculation of $3 \times 10^{6}$ tumor cells into C57BL/6 mice, the growth rate of LLC tumors treated by PEGylated NE was slower than those tumors treated by LBT solution, blank NE, and normal saline (Figure 7). The proliferation rate of LLC cells in the mice treated by LBT-OA-PEG-NE was obviously lower than that in the mice treated by other formulations. According to Figure 7, the curve of LBT-OA-PEG-NE was the lowest all the time, while the curves of blank NE and saline were both the highest at the same time. First, it showed the antitumor effect of the LBT-OA-PEG-NE was much better than that of LBT solution. Secondly, they both had an antitumor effect on this model, while the blank NE and saline had no effect. The observed differences in the growth of LLC tumors were due to the prolongated circulation of the PEGylated NE. In addition, the observed differences in the tumors' growth of different formulations also existed in survival curves. Our analysis showed that LBT-OA-PEGNE could prolongate mean survival time to 21.875 days, which was greater than that for the mice treated with blank NE, saline's about 12 days, and the mice treated with the LBT solution about 15 days (Figure 8). Further, the similar tendency of blank NE and saline showed the excipients

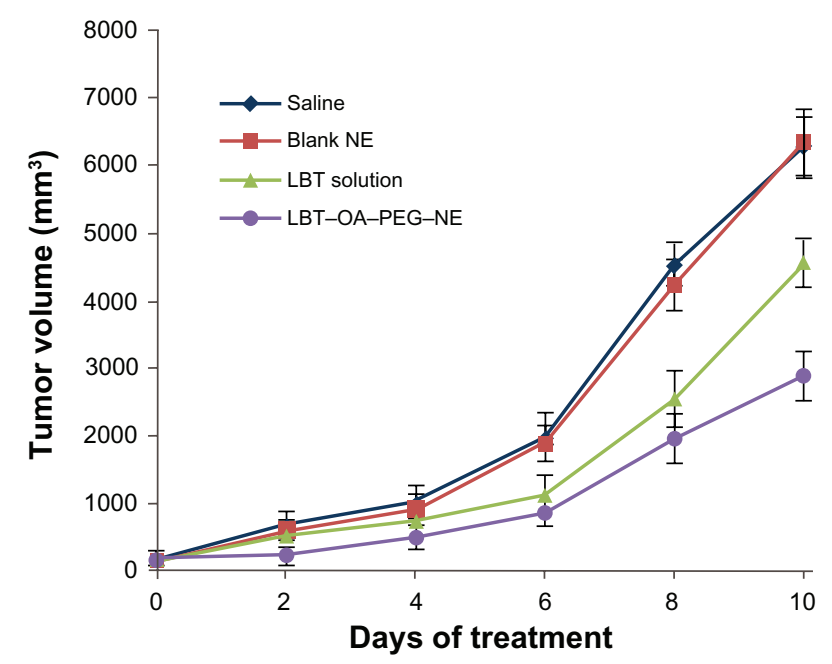

Figure 7 Volume of LLC tumor treated by saline, blank nanoemulsions, LBT-OANE, and LBT-OA-PEG-NE.

Abbreviations: LBT, lycobetaine; LBT-OA-NE, nanoemulsion loaded with lycobetaine-oleic acid complex; LBT-OA-PEG-NE, PEGylated LBT-OA-NE; LLC, Lewis lung carcinoma cell line; NE, nanoemulsion. 


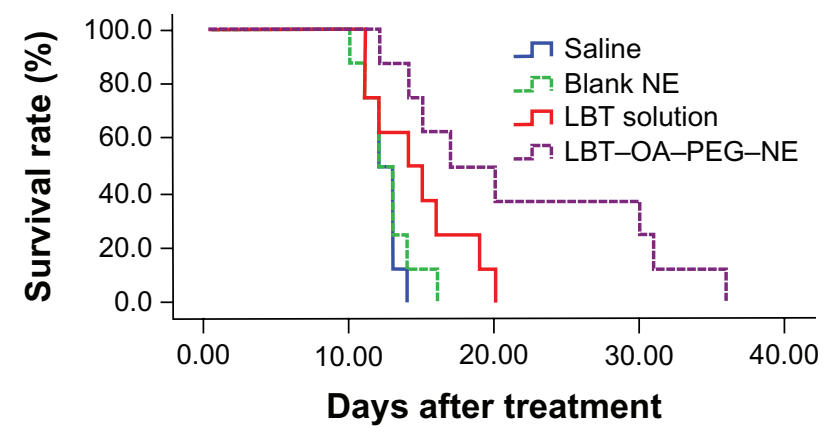

Figure 8 Life-prolonging effect of LBT solution, LBT-OA-PEG-NE and the control group (saline and blank nanoemulsions).

Notes: Life-prolonging effect was determined from the survival time (day) of LLCtumor-bearing mice. Survival curves of saline (line, blue), blank nanoemulsions (dotted, green), $10 \mathrm{mg} / \mathrm{kg}$ LBT solution-treated (line, red), and LBT-OA-PEG-NE (dotted, purple) groups are shown. Eight animals were in each treated group. Abbreviations: LBT, lycobetaine; LBT-OA-NE, nanoemulsion loaded with lycobetaine-oleic acid complex; LBT-OA-PEG-NE, PEGylated LBT-OA-NE; LLC, Lewis lung carcinoma cell line; NE, nanoemulsion.

didn't have antitumor activity. Thus, the differences in the growth rate of LLC tumors and the survival time were due to LBT's antitumor effect. Indeed, after an injection of LBT solution, the tail began to fester as a side effect (Figure 9). It was probably caused by the acetic acid, which was added to make the LBT dissolve in the water.

\section{Antitumor activity in lung metastasis model}

Although the PEGylated NE inhibition of tumor was proved in the former heterotopic model, effect on lung metastasis model seemed to make more sense. In that experiment, we tested whether lung metastasis tumor could be prevented by LBT-OA-PEG-NE. When LBT-OA-PEG-NE treatment was given to mice bearing B16F10 tumor, a longer prolonged survival time was observed (Figure 10). As shown in Figure 10, LBT-OA-PEG-NE treatment resulted in significant inhibition of B16F10. Mean survival time of PEGylated NE extended to 45 days, whereas the mean time of control groups (blank NE and saline) and LBT solution was about 23 days and 31 days, respectively. Again, we

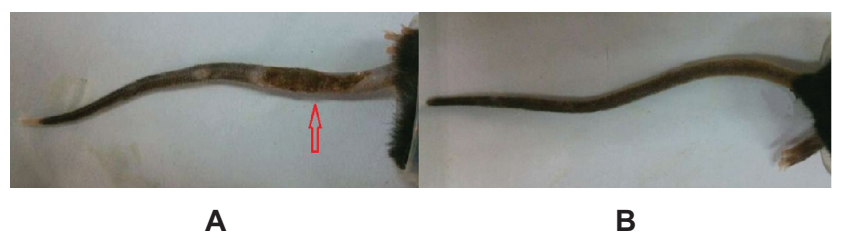

Figure 9 Tail skin ulcer as a side effect of LBT solution. (A) was treated by LBT solution, and the fester was marked by a red arrow. (B) was treated by LBT-OAPEG-NE, and no fester was observed.

Abbreviations: LBT, lycobetaine; LBT-OA-NE, nanoemulsion loaded with lycobetaine-oleic acid complex; LBT-OA-PEG-NE, PEGylated LBT-OA-NE; NE, nanoemulsion.

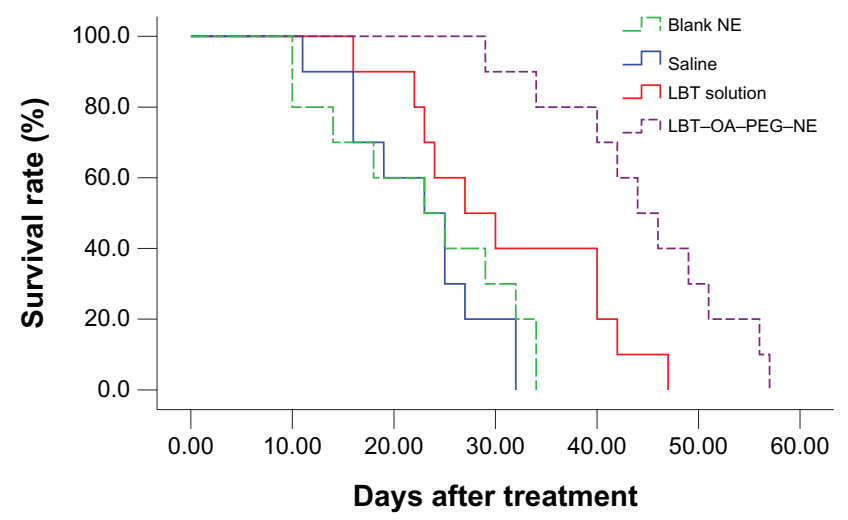

Figure 10 Life-prolonging effect of LBT solution, LBT-OA-PEG-NE and the control group (saline and blank nanoemulsions).

Notes: Life-prolonging effect was determined from the survival time (day) of BI6FI0-tumor-bearing mice. Survival curves of saline, blank nanoemulsions, $10 \mathrm{mg} / \mathrm{kg}$ LBT solution-treated, and LBT-OA-PEG-NE groups are shown. Ten animals were in each treated group.

Abbreviations: LBT, lycobetaine; LBT-OA-NE, nanoemulsion loaded with lycobetaine-oleic acid complex; LBT-OA-PEG-NE, PEGylated LBT-OA-NE; NE, nanoemulsion.

found that the LBT solution treatment resulted in very weak tumor inhibition. But this inhibition of the B16F10 tumor in mice was less profound than that in NE-treated mice, and the side effect of fester in the tail was also observed. These results indicate that the PEGylated NE could potentiate the therapeutic efficacy of LBT.

\section{Conclusion}

Overall, the LBT-OA-PEG-NE showed improved pharmacokinetic and altered tissue distribution profiles, which could be related to the enhanced lipophilicity of LBT, the shielding effect of PEG chains and the prolonged drug release. The present study demonstrated that a novel LBT-OA ionic complex was prepared to alter the solubility of LBT and entrap LBT into lipid NE. The method of preparing the ionic complex with OA develops a new prospective for carrying positively charged drugs into lipid-based colloidal drug carriers. In addition, the HPH method, feasible for scale-up production, allowed instantaneous and reproducible formation of LBT-OA-PEG-NE, with a diameter value, small PDI, high entrapment efficiency, and improved release properties. Most importantly, all the used materials were approved for intravenous injection, so the preparation has a great potential for clinical application. Pharmacokinetics and biodistribution studies in rats showed that LBT-OA-PEG-NE demonstrated higher LBT levels in blood and longer circulation time than free LBT, which suggested that the LBT-OA-PEG-NE may exhibit better therapeutic efficacy. LBT-OA-PEG-NE significantly decreased LBT concentration in the heart, liver, and kidney. Moreover, the LBT-OA-PEG-NE exhibited higher growth inhibitory effect 
and longer survival time than free LBT in both heterotopic and lung metastasis tumor models. Hence, this novel formulation has a promising potential as an alternative parenteral colloidal delivery system of LBT for cancer treatment.

\section{Acknowledgments}

This work was funded by the National Basic Research Programs of the People's Republic of China (973 program; Number: 2013CB932504) and supported by the program for National S \& T Major Project of the People's Republic of China (2012ZX09304004).

\section{Disclosure}

The authors report no conflicts of interest in this work.

\section{References}

1. Weng ZY, Wang ZY, Yan XM. A new antitumour agent ungeremine (AT-1840) and its structure-activity relationship. Yao Xиe Xиe Bao. 1982;17(10):744-749. Chinese [with English abstract].

2. Ghosal S, Singh SK, KumarY, Unnikrishnan S, Chattopadhyay S. The role of ungeremine in the growth-inhibiting and cytotoxic effects of lycorine: evidence and speculation. Planta Med. 1988;54(2): 114-116.

3. Zhang SY, Lu FL, Yang JL, Wang LJ, Xu B. Effect on animal tumors and toxicity of lycobetaine acetate (author's translation). Zhongguo Yao Li Xue Bao. 1981;2(1):41-45. Chinese [with English abstract].

4. Barthelmes HU, Niederberger E, Roth T, et al. Lycobetaine acts as a selective topoisomerase II beta poison and inhibits the growth of human tumor cells. Br J Cancer. 2001;85(10):1585-1591.

5. Casu L, Cottiglia F, Leonti M, et al. Ungeremine effectively targets mammalian as well as bacterial type I and type II topoisomerases. Bioorg Med Chem Lett. 2011;21(23):7041-7044.

6. Wu Z, Chen Y, Feng X, Xia B. Pharmacology study of medicinal plant alkaloids of Amaryllidaceae medicinal plants. Chinese Wild Plant Resources. 2008;27(5):26-31.

7. Qinghe H, et al. Lycobetaine (AT-1840) in the comprehensive treatment of gynecological late malignant tumor - with 43 cases summarized. Zhejiang combine traditional Chinese and western medicine magazine. 1997;7(3):3. Available at http://www.cqvip.com/ qk/98552x/199703/2474273.html. Chinese.

8. Liang G, Xu Y, Xu B. Pharmacokinetics of ungeremine acetate in rats. Chinese Pharmacological Bulletin. 1997;13(1):3. Available at http://en.cnki.com.cn/Article_en/CJFDTOTAL-YAOL701.011.htm. Chinese.

9. Fernández-Campos F, Clares NB, López SO, Alonso MC, Calpena Campmany AC. Evaluation of novel nystatin nanoemulsion for skin candidosis infections. Mycoses. 2013;56(1):70-81.

10. Xiang QY, Wang MT, Chen F, et al. Lung-targeting delivery of dexamethasone acetate-loaded solid lipid nanoparticles. Arch Pharm Res. 2007;30(4):519-525.

11. Müller RH, Shegokar R, Keck CM. Twenty years of lipid nanoparticles (SLN and NLC): present state of development and industrial applications. Curr Drug Discov Technol. 2011;8(3):207-227.

12. Divsalar A, Saboury AA, Nabiuni M, Zare Z, Kefayati ME, Seyedarabi A. Characterization and side effect analysis of a newly designed nanoemulsion targeting human serum albumin for drug delivery. Colloids Surf B Biointerfaces. 2012;98:80-84.

13. Storm G, Belliot S, Daemen T, Lasic D. Surface modification of nanoparticles to oppose uptake by the mononuclear phagocyte system. $A d v$ Drug Deliv Rev. 1995;17(1):31-48.
14. SadzukaY, NakadeA, Hirama R, et al. Effects of mixed polyethyleneglycol modification on fixed aqueous layer thickness and antitumor activity of doxorubicin containing liposome. Int J Pharm. 2002;238(1-2): 171-180.

15. Siddiqui A, Gupta V, Liu YY, Nazzal S. Doxorubicin and MBO-asGCS oligonucleotide-loaded lipid nanoparticles overcome multidrug resistance in adriamycin-resistant ovarian cancer cells (NCI/ADR-RES). Int J Pharm. 2012;431(1-2):222-229.

16. Higashiyama M, Inada K, Ohtori A, Kakehi K. NMR analysis of ion-pair formation between timolol and sorbic acid in ophthalmic preparations. J Pharm Biomed Anal. 2007;43(4):1335-1342.

17. Ma P, Dong X, Swadley CL, et al. Development of idarubicin and doxorubicin solid lipid nanoparticles to overcome Pgp-mediated multiple drug resistance in leukemia. J Biomed Nanotechnol. 2009;5(2): $151-161$.

18. Zhang X, Sun X, Li J, Zhang X, Gong T, Zhang Z. Lipid nanoemulsions loaded with doxorubicin-oleic acid ionic complex: characterization, in vitro, and in vivo studies. Pharmazie. 2011;66(7):496-505.

19. Pontes-Arruda A. Biological benefits of an oleic acid-rich lipid emulsion for parenteral nutrition. Clinical Nutrition Supplements. 2009;4(1):19-23.

20. Olbrich C, Gessner A, Kayser O, Müller RH. Lipid-drug-conjugate (LDC) nanoparticles as novel carrier system for the hydrophilic antitrypanosomal drug diminazenediaceturate. J Drug Target. 2002;10(5): 387-396.

21. Olbrich C, Gessner A, Schröder W, Kayser O, Müller RH. Lipid-drug conjugate nanoparticles of the hydrophilic drug diminazene-cytotoxicity testing and mouse serum adsorption. J Control Release. 2004;96(3): 425-435.

22. Quintanar-Guerrero D, Allémann E, Fessi H, Doelker E. Applications of the ion-pair concept to hydrophilic substances with special emphasis on peptides. Pharm Res. 1997;14(2):119-127.

23. Kalinkova GN. Complexation: Noncyclodextrins. In: Swarbrick J, editor. Encyclopedia of Pharmaceutical Technology, 3rd ed. New York: Informa Healthcare USA Inc; 2006:697-706.

24. Kanjilal S, Kaki SS, Rao BV, et al. Hypocholesterolemic effects of low-calorie structured lipids on rats and rabbits fed on normal and atherogenic diet. Food Chem. 2013;136(1):259-265.

25. Wabel C. Influence of Lecithin on Structure and Stability of Parenteral Fat Emulsions [doctoral dissertation]. Frankfurt, Alemania: University of Erlangen-Nuremberg; 1998.

26. Zhao D, Gong T, Fu Y, et al. Lyophilized Cheliensisin A submicron emulsion for intravenous injection: characterization, in vitro, and in vivo antitumor effect. Int J Pharm. 2008;357(1-2):139-147.

27. Zhao Y, Wang L, Yan M, et al. Repeated injection of PEGylated solid lipid nanoparticles induces accelerated blood clearance in mice and beagles. Int J Nanomedicine. 2012;7:2891-2900.

28. Sriwongsitanont S, Ueno M. Effect of a PEG lipid (DSPE-PEG2000) and freeze-thawing process on phospholipid vesicle size and lamellarity. Colloid Polym Sci. 2004;282(7):753-760.

29. Needham D, Stoicheva N, Zhelev DV. Exchange of monooleoylphosphatidylcholine as monomer and micelle with membranes containing poly(ethylene glycol)-lipid. Biophys J. 1997;73(5): 2615-2629.

30. Siepmann J, Peppas NA. Modeling of drug release from delivery systems based on hydroxypropyl methylcellulose (HPMC). Adv Drug Deliv Rev. 2001;48(2-3):139-157.

31. Zhang Y, Huo M, Zhou J, et al. DDSolver: an add-in program for modeling and comparison of drug dissolution profiles. AAPS J. 2010;12(3): 263-271.

32. Moghimi SM, Hunter AC, Murray JC. Long-circulating and targetspecific nanoparticles: theory to practice. Pharmacol Rev. 2001;53(2): 283-318.

33. Vonarbourg A, Passirani C, Saulnier P, Benoit JP. Parameters influencing the stealthiness of colloidal drug delivery systems. Biomaterials. 2006;27(24):4356-4373. 
34. Vonarbourg A, Passirani C, Saulnier P, Simard P, Leroux JC, Benoit JP. Evaluation of pegylated lipid nanocapsules versus complement system activation and macrophage uptake. J Biomed Mater Res A. 2006;78(3): $620-628$.

35. Mosqueira VC, Legrand P, Gulik A, et al. Relationship between complement activation, cellular uptake, and surface physicochemical aspects of novel PEG-modified nanocapsules. Biomaterials. 2001;22(22): 2967-2979.
36. Moghimi SM, Szebeni J. Stealth liposomes and long-circulating nanoparticles: critical issues in pharmacokinetics, opsonization and protein-binding properties. Prog Lipid Res. 2003;42(6):463-478.

International Journal of Nanomedicine

\section{Publish your work in this journal}

The International Journal of Nanomedicine is an international, peerreviewed journal focusing on the application of nanotechnology in diagnostics, therapeutics, and drug delivery systems throughout the biomedical field. This journal is indexed on PubMed Central, MedLine, CAS, SciSearch $\AA$, Current Contents ${ } /$ Clinical Medicine,
Journal Citation Reports/Science Edition, EMBase, Scopus and the Elsevier Bibliographic databases. The manuscript management system is completely online and includes a very quick and fair peer-review system, which is all easy to use. Visit http://www.dovepress.com/ testimonials.php to read real quotes from published authors.

Submit your manuscript here: http://www.dovepress.com/international-journal-of-nanomedicine-journal 